\title{
Concordancia diagnóstica entre la broncoscopia y la histopatología en pacientes con neoplasias pulmonares
}

\author{
Ricardo Durán-Acuña,* Carolina Araque,* Juan José Chaves, ${ }^{\ddagger}$ Julián \\ Jiménez, ${ }^{\S}$ Fernando Polo," $\bowtie$ Rafael Parra-Medina ${ }^{\uparrow, \|}$
}

\begin{abstract}
*Departamento de Medicina Interna-Neumología. Fundación Universitaria de Ciencias de la Salud, Hospital de San José, Bogotá, Colombia.
łSemilleros de Investigación de Patología. Fundación Universitaria de Ciencias de la Salud. Bogotá, Colombia.

${ }_{\S}^{\S}$ Departamento de Cirugía General. Fundación Universitaria de Ciencias de la Salud. Bogotá, Colombia.

"Departamento de Patología. Fundación Universitaria de Ciencias de la Salud, Bogotá, Colombia.

"Instituto de Investigación. Fundación Universitaria de Ciencias de la Salud, Bogotá, Colombia.
\end{abstract}

\begin{abstract}
RESUMEN. Antecedentes: El cáncer de pulmón es una de las principales causas de muerte a nivel mundial, cuando se descubre generalmente se hace en estadíos avanzados, constituyendo un mal pronóstico. Este último mejora con un diagnóstico temprano y manejo oportuno. El objetivo de este estudio es evaluar la concordancia entre los hallazgos fibrobroncoscópicos e histopatológicos en pacientes con neoplasias pulmonares, y de esta manera conocer si existe la viabilidad de realizar estudios complementarios de inmunohistoquímica o pruebas moleculares a los casos sospechosos de malignidad en la fibrobroncoscopia. Materiales y métodos: Se realizó un estudio de concordancia diagnóstica incluyendo pacientes mayores de 18 años con diagnóstico de neoplasias pulmonares. Se estimó el acuerdo absoluto y el acuerdo más allá del azar mediante el estadístico de concordancia de Kappa de Cohen. Resultados: Se incluyeron en el estudio 369 casos, de los cuales 207 pacientes (56.1\%) fueron de sexo masculino. La mediana de la edad fue de 58 años (RIQ 23). La concordancia del diagnóstico fibrobroncoscópico e histopatológico de neoplasia pulmonar fue moderada con un índice de Kappa positivo de 0.605 (IC95\% de 0.496-0.715). La tasa de acuerdo fue del 89\%. El adenocarcinoma fue el tumor que mostró menor acuerdo entre las dos pruebas. Conclusiones: El presente estudio mostró una concordancia moderada entre los hallazgos fibrobroncoscópicos e histopatológicos. Estos hallazgos confirman que la sospecha en la endoscopia es fundamental para encaminar el diagnóstico y solicitar pruebas preliminares para un adecuado y oportuno estudio histológico.
\end{abstract}

Palabras clave: Neoplasia pulmonar, cáncer, fibrobroncoscopia, biopsia, concordancia diagnóstica.

\section{Diagnostic concordance between bronchoscopy and histopathology in patients with pulmonary neoplasms}

ABSTRACT. Background: Lung cancer is one of the leading causes of death worldwide, it is generally discovered in advanced stages, constituting a poor prognosis, which this last one has an improvement with early diagnosis and timely management. The aim of this study is evaluate the concordance between the fibrobronchoscopic and histopathological findings in patients with pulmonary neoplasias, in order to perform complementary studies of immunohistochemistry or molecular tests to suspected cases of malignancy in the fibrobronchoscopic. Materials and methods: A diagnostic agreement study was carried out, including patients older than 18 years with a diagnosis of pulmonary neoplasms. Absolute agreement and agreement beyond chance were estimated using Cohen's Kappa concordance statistic. Results: 369 cases were included in the study, of which 207 patients (56.1\%) were male. The median of age was 58 years (IQR 23). The concordance of the fibrobronchoscopic and histopathological diagnosis of pulmonary neoplasia was moderate with a positive Kappa index of 0.605 (95\% $\mathrm{Cl}$ of $0.496-0.715)$. The agreement rate was $89 \%$. The adenocarcinoma was the tumor that showed the least agreement between the two tests. Conclusion: The present study showed a moderate concordance between the fibrobronchoscopic and histopathological findings, with an agreement rate of $89 \%$. These findings confirm that the suspicion in endoscopy is fundamental to guide the diagnosis and request preliminary tests for an appropriate and timely histological study.

Key words: Pulmonary neoplasia, cancer, fibrobronchoscopy, biopsy, diagnostic concordance.

\section{INTRODUCCIÓN}

\section{$\square$ Autor para correspondencia:}

Dr. José Fernando Polo Nieto, Grupo de Patología-Fundación Universitaria de Ciencias de la Salud. Bogotá D.C., Colombia. Correo electrónico: jfpolo@fucsalud.edu.co

Trabajo recibido: 22-X-2018; aceptado: 08-IV-2019
El cáncer de pulmón es una de las principales causas de morbimortalidad en hombres y mujeres alrededor del mundo. ${ }^{1-4}$ En el 2012 se consideró la primera causa de morbimortalidad en países desarrollados y la segunda causa en países en vía de desarrollo. ${ }^{2}$ Su incidencia ha 
aumentado progresivamente en los últimos años; y llamativamente se ha incrementado en mujeres, llegando a ser su prevalencia muy similar a la de los hombres. ${ }^{4}$

En la mayoría de pacientes el diagnóstico se retrasa debido a la ausencia de signos específicos; siendo el 90\% de los pacientes asintomáticos en estadío temprano. Es sólo cuando la enfermedad es metastásica o está localmente avanzada que presentan sintomatología. Los síntomas más frecuentes son tos, hemoptisis, disnea y fatiga. ${ }^{3,5}$

Si las neoplasias pulmonares son diagnosticadas temprano y tratadas precozmente, el pronóstico mejora de manera considerable. ${ }^{5,6}$ Por lo anterior, es importante usar las múltiples ayudas diagnósticas disponibles en pacientes con sospecha clínica y con lesiones sugestivas de malignidad o de alto riesgo para desarrollar neoplasias pulmonares. Para el estudio clínico en estos pacientes comúnmente se inicia con métodos mínimamente invasivos como la radiografía de tórax y/o la TAC de tórax, y según los hallazgos radiológicos se determina qué pacientes son candidatos a fibrobroncoscopia para toma de muestras de tejido y su estudio histopatológico posterior.

La fibrobroncoscopia flexible es un estudio invasivo que permite la visualización de la vía aérea y la toma de muestras de tejido por medio de lavado broncoalveolar, cepillado bronquial o biopsia transbronquial. ${ }^{5,7-12} \mathrm{En} \mathrm{la}$ actualidad se están desarrollando métodos innovadores fibrobroncoscópicos para la detección temprana de cáncer de pulmón.

En el estudio histopatológico, en las biopsias pulmonares por lo común se deben ampliar los estudios con marcadores de inmunohistoquímica para definir las características y el subtipo histológico de los tumores; y con esto definir los estudios moleculares que permitan precisar exactamente de qué tipo de tumor se trata y de esta manera encaminar el tratamiento dirigido. ${ }^{13,14}$ Cabe destacar que en algunas oportunidades no es posible realizar estos estudios adicionales debido a trámites administrativos, no disponibilidad de las pruebas, materiales insuficientes o material inadecuado. Por tal motivo, el objetivo del presente estudio es evaluar la concordancia entre los hallazgos fibrobroncoscópicos e histopatológicos en pacientes con neoplasias pulmonares para conocer si existe la viabilidad de realizar estudios complementarios de inmunohistoquímica o pruebas moleculares a los casos sospechosos de malignidad en la fibrobroncoscopia.

\section{MATERIALES Y MÉTODOS}

Diseño: se trata de un estudio de concordancia diagnóstica. Población y muestra: se incluyeron pacientes mayores de 18 años en los que se realizó fibrobroncoscopia con biopsia en el Servicio de Neumología del Hospital San José de Bogotá, Colombia, entre enero 2014 y octubre de 2017. Para el cálculo de tamaño de muestra se empleó el método desarrollado por Donner et al. ${ }^{15}$ quienes desarrollaron el cálculo para el estadístico kappa en el caso de 2 evaluadores o dos métodos de evaluación de una variable nominal binaria. Los datos de entrada para el cálculo fueron los siguientes: error tipo I (alfa) de 0.05, error tipo II (beta) de 0.2, probabilidad de clasificación correcta 0.9 , donde este dato fue obtenido a partir de la prueba piloto realizada con el $10 \%$ de una base de datos existente en el Servicio de Patología y Neumología del Hospital de San José, en el que se documentó un $90 \%$ de acuerdo entre los observadores. Como hipótesis nula $(\mathrm{HO})$ se definió un kappa de 0.4, puesto que según la clasificación de Landis et al. ${ }^{16}$ este valor corresponde al límite superior de un nivel de concordancia leve; y como hipótesis alterna $(\mathrm{H} 1)$ un kappa mayor de 0.61 , puesto que según la misma clasificación este valor corresponde al límite inferior de un acuerdo sustancial o alto. Con base en los anteriores supuestos se obtuvo un cálculo de tamaño de muestra de 369 casos.

Se utilizó un instrumento de recolección electrónico diseñado por los investigadores en formato Excel v2012, que incluyó información sociodemográfica, indicaciones de fibrobroncoscopia, hallazgos fibrobroncoscópicos y resultados del estudio histopatológico. Estos datos fueron extraídos de los reportes de la fibrobroncoscopia por parte del Servicio de Neumología y los datos de Patología de la base de datos de este servicio. El análisis estadístico fue realizado por medio del software Stata V.13 ${ }^{\circledR}$. El estudio fue aprobado por el Comité de Investigaciones y Ética de la Fundación Universitaria de Ciencias de la Salud.

Interpretación de la broncoscopia e histopatología. El estudio fibrobroncoscópico de todos los pacientes fue realizado por el mismo neumólogo con 20 años de experiencia (RD). El equipo que se utilizó para la prueba fue un fibrobroncoscopio marca Olympus, el cual tiene mantenimiento y calibración según protocolos del hospital. Se consideró como hallazgos sospechosos de malignidad la presencia de estrías hipertróficas, masa, empedrado e hipervascularización. También se evaluaron otras variables como endobronquitis antracótica, compresión extrínseca, disminución del calibre, anillos fibróticos, endobronquitis purulenta, fosetas bronquiales, entre otras.

El estudio histopatológico de todos los pacientes fue desarrollado por el mismo patólogo con 10 años de experiencia (FP). La aproximación inicial de conocer si la biopsia era una lesión tumoral o no tumoral fue 
realizada sin conocer el reporte fibrobroncoscópico. Posteriormente, el patólogo para lograr el diagnóstico definitivo utilizó datos de historia clínica, hallazgos de la fibrobroncoscopia, de las imágenes radiológicas (radiografía de tórax y tomografía axial computarizada) y ayudas complementarias histopatológicas como inmunohistoquímica o coloraciones de histopatología, lo cual fue realizado según los criterios de la Organización Mundial de la Salud. ${ }^{17}$

Análisis estadístico. Se evaluó la concordancia entre el diagnóstico fibrobroncoscópico de neoplasias pulmonares y los hallazgos histopatológicos, utilizando un coeficiente de Kappa de Cohen, al ser una variable cualitativa nominal dicotómica. El acuerdo entre los observadores se calculó basado en una tabla de contingencia de dos vías entre las observaciones obtenidas en fibrobroncoscopia e histopatología en la que se consideraron resultados positivos y negativos para cada una de las pruebas. El acuerdo absoluto se calculó sumando los resultados positivos para las dos pruebas y los resultados negativos para las dos pruebas, dividido sobre el total de estudios realizados. Para conocer el acuerdo más allá del azar, se calculó el coeficiente Kappa de Cohen, en donde un valor de 0 fue interpretado como un acuerdo igual al esperado por azar y valores superiores a 0 con resultados de $p<0.05$ fueron considerados estadísticamente significativos. Con el objetivo de darle sentido clínico a los hallazgos, los resultados se interpretaron con base en la escala propuesta por Landis et al. ${ }^{16}$ en la que valores por debajo de 0 fueron considerados sin acuerdo para neoplasia, valores entre $0.00-0.20$ con acuerdo insignificante (pobre), entre 0.21 y 0.40 acuerdo discreto (débil), de 0.41 a 0.60 moderado, 0.61-0.80 acuerdo bueno (sustancial), y mayor a 0.81 acuerdo casi perfecto (muy bueno). El coeficiente Kappa fue reportado con intervalos de confianza del 95\%.

\section{RESULTADOS}

Características generales. Se incluyeron 369 casos en los cuales se realizó fibrobroncoscopia y toma de biopsia con estudio histopatológico, 162 pacientes (43.9\%) fueron de sexo femenino, y 207 (56.1\%) de sexo masculino. La mediana de edad fue de 58 años con un rango intercuartílico de 23.

Indicaciones para broncoscopia. Las principales indicaciones para realización de fibrobroncoscopia y biopsia fueron: sospecha de tuberculosis (17.3\%), sospecha de neoplasia (16.8\%), enfermedad pulmonar intersticial difusa (EPID [16.3\%]), neumonía (8.9\%), masa pulmonar (8.7\%), atelectasia (6.2\%), sospecha de metástasis de otro tumor primario (5.4\%) y hemoptisis (2.7\%).

Hallazgos broncoscópicos. En el procedimiento endoscópico (tabla 1), en 61 casos (16.5\%) se encontraron hallazgos compatibles con infiltración neoplásica, mientras 118 (32.3\%) mostraron endobronquitis antracótica, 137 (37.1\%) fueron endoscópicamente normales, $8(2.2 \%)$ evidenciaron compresión extrínseca, 5 (1.4\%) disminución del calibre, 2 (0.5\%) casos con anillos fibróticos, $2(0.5 \%)$ casos de candidiasis, 6 (1.6\%) con endobronquitis purulenta, $5(1.4 \%)$ con fosetas bronquiales, $2(0.5 \%)$ con hemorragia alveolar y otros hallazgos 23 casos (6.2\%).

Hallazgos histopatológicos. En 64 pacientes se reconoció tumor maligno (17.3\% [tabla 1]). El 39.1\% eran adenocarcinoma pulmonar primario, 28.1\% carcinoma de célula escamosa, tumor neuroendocrino en $9.4 \%$, tumor de células pequeñas $7.8 \%$, linfoma en $6.3 \%$, lesiones metastásicas de origen ginecológico en el $7.8 \%$ y un caso de sarcoma (mujer de 70 años).

Se encontraron 305 casos negativos para neoplasia (82.7\%), donde los principales hallazgos fueron: fibroantracosis (21.3\%), histología usual (15.1\%), bronquitis crónica (13.4\%), infiltrado crónico inespecífico (12.8\%), metaplasia escamosa (4.6\%), hemorragia alveolar (3.9\%), entre otros (tabla 1). Se reconocieron casos de enfermedades infecciosas como neumonía bacteriana en 12 casos, infección por tuberculosis se encontró en 17 casos, un caso de criptococosis y un caso de Pneumocystis jirovecii.

Acuerdo diagnóstico entre la broncoscopia y la histopatología. La tasa de acuerdo fue del $89 \%$, con un índice de acuerdo moderado (Kappa $=0.605$ (IC95\% de 0.496-0.715). Cuarenta y dos casos fueron positivos para neoplasia pulmonar en la broncoscopia y en biopsia, y 286 casos fueron negativos en broncoscopia y en biopsia. De los casos con sospecha de malignidad en la broncoscopia y negativos histológicamente $(n=19)$ se reconocieron 5 casos con infiltrado crónico inespecífico, 6 casos con otros hallazgos inespecíficos, 3 casos de histología usual, 3 casos de metaplasia escamosa, 1 caso de bronquitis crónica y 1 caso de tuberculosis (tabla 1). De los 22 casos negativos en la broncoscopia para malignidad pero positivos histológicamente, 11 casos correspondían a adenocarcinoma primario de pulmón, 4 a linfomas, 2 a carcinoma de células pequeñas, 3 casos a metástasis, 1 caso a carcinoma de célula escamosa y el restante a carcinoma neuroendocrino (tabla 1). Los hallazgos broncoscópicos de estos pacientes fueron endobronquitis antracótica, sin alteraciones en la broncoscopia, compresión extrínseca y disminución del calibre (tabla 2). 
Tabla 1: Características de pacientes con estudio de enfermedad pulmonar por broncoscopia.

\begin{tabular}{|c|c|c|c|c|c|}
\hline & \multicolumn{5}{|c|}{ Broncoscopia/biopsia } \\
\hline & $\begin{array}{l}\text { Positivo/ } \\
\text { positivo }\end{array}$ & $\begin{array}{l}\text { Positivo/ } \\
\text { negativo }\end{array}$ & $\begin{array}{l}\text { Negativo/ } \\
\text { positivo }\end{array}$ & $\begin{array}{c}\text { Negativo/ } \\
\text { negativo }\end{array}$ & Total n (\%) \\
\hline \multicolumn{6}{|l|}{ Características } \\
\hline Pacientes & 42 & 19 & 22 & 286 & 369 \\
\hline Hombres & 19 & 12 & 10 & 166 & 207 \\
\hline Mujeres & 23 & 7 & 12 & 120 & 162 \\
\hline \multicolumn{6}{|l|}{ Hallazgos broncoscópicos } \\
\hline Infiltración neoplásica & 42 & 19 & 0 & 0 & $61(16.5)$ \\
\hline Endobronquitis antracótica & 0 & 0 & 11 & 107 & $118(32.3)$ \\
\hline Sin alteraciones & 0 & 0 & 8 & 129 & $137(37.1)$ \\
\hline Compresión extrínseca & 0 & 0 & 2 & 6 & $8(2.2)$ \\
\hline Disminución del calibre & 0 & 0 & 1 & 4 & $5(1.4)$ \\
\hline Anillos fibróticos & 0 & 0 & 0 & 2 & $2(0.5)$ \\
\hline Candidiasis & 0 & 0 & 0 & 2 & $2(0.5)$ \\
\hline Endobronquitis purulenta & 0 & 0 & 0 & 6 & $6(1.6)$ \\
\hline Fosetas bronquiales & 0 & 0 & 0 & 5 & $5(1.4)$ \\
\hline Hemorragia alveolar & 0 & 0 & 0 & 2 & $2(0.5)$ \\
\hline Otros hallazgos & 0 & 0 & 0 & 23 & $23(6.2)$ \\
\hline \multicolumn{6}{|l|}{ Diagnóstico histopatológico } \\
\hline \multicolumn{5}{|l|}{ Lesión tumoral maligna } & $64(17.3)$ \\
\hline Adenocarcinoma pulmonar primario & 14 & 0 & 11 & 0 & $25(39.1)$ \\
\hline Carcinoma de célula escamosa & 17 & 0 & 1 & 0 & $18(28.1)$ \\
\hline Tumor neuroendocrino & 5 & 0 & 1 & 0 & $6(9.4)$ \\
\hline Carcinoma de células pequeñas & 3 & 0 & 2 & 0 & $5(7.8)$ \\
\hline Linfoma & 0 & 0 & 4 & 0 & $4(6.3)$ \\
\hline Metástasis de origen ginecológico & 2 & 0 & 3 & 0 & $5(7.8)$ \\
\hline Sarcoma & 1 & 0 & 0 & 0 & $1(1.6)$ \\
\hline \multicolumn{5}{|l|}{ Hallazgos no tumorales malignos } & $305(82.7 \%)$ \\
\hline Fibroantracosis & 0 & 0 & 0 & 65 & $65(21.3)$ \\
\hline Histología usual & 0 & 3 & 0 & 43 & $46(15.1)$ \\
\hline Bronquitis crónica & 0 & 1 & 0 & 40 & $41(13.4)$ \\
\hline Infiltrado crónico inespecífico & 0 & 5 & 0 & 34 & $39(12.8)$ \\
\hline Metaplasia escamosa & 0 & 3 & 0 & 11 & $14(4.6)$ \\
\hline Hemorragia alveolar & 0 & 0 & 0 & 12 & $12(3.9)$ \\
\hline Neumonía bacteriana & 0 & 0 & 0 & 12 & $12(3.9)$ \\
\hline Neumonitis intersticial & 0 & 0 & 0 & 13 & $13(4.3)$ \\
\hline Neumonía en organización & 0 & 0 & 0 & 6 & $6(2)$ \\
\hline Neumonitis de hipersensibilidad & 0 & 0 & 0 & 5 & $5(1.6)$ \\
\hline Papiloma & 0 & 0 & 0 & 1 & $1(0.3)$ \\
\hline
\end{tabular}


Continúa Tabla 1: Características de pacientes con estudio de enfermedad pulmonar por broncoscopia.

\begin{tabular}{|l|c|c|c|c|c|}
\hline \multirow{2}{*}{} & \multicolumn{5}{|c|}{ Broncoscopia/biopsia } \\
\cline { 2 - 6 } & $\begin{array}{c}\text { Positivo/ } \\
\text { positivo }\end{array}$ & $\begin{array}{c}\text { Positivo/ } \\
\text { negativo }\end{array}$ & $\begin{array}{c}\text { Negativo/ } \\
\text { positivo }\end{array}$ & $\begin{array}{c}\text { Negativo/ } \\
\text { negativo }\end{array}$ & 16 \\
\hline Tuberculosis & 0 & 1 & 0 & 4 \\
\hline Sarcoidosis & 0 & 0 & 0 & $4(1.3)$ \\
\hline Criptococosis & 0 & 0 & 0 & $1(0.3)$ \\
\hline Pneumocystis jirovecii & 0 & 0 & 0 & 1 \\
\hline Eosinofilia & 0 & 0 & 0 & $1(0.3)$ \\
\hline Otros hallazgos inespecíficos & 0 & 6 & 0 & 1 \\
\hline
\end{tabular}

Tabla 2: Hallazgos broncoscópicos y diagnóstico histopatológico de pacientes con broncoscopia negativa y biopsias positivas para malignidad.

\begin{tabular}{|c|c|c|}
\hline $\begin{array}{l}\text { Diagnóstico } \\
\text { broncoscópico }\end{array}$ & Diagnóstico histopatológico & $n=22$ \\
\hline \multirow{4}{*}{$\begin{array}{l}\text { Endobronquitis } \\
\text { antracótica }\end{array}$} & $\begin{array}{l}\text { Adenocarcinoma pulmonar } \\
\text { primario }\end{array}$ & 8 \\
\hline & Carcinoma de célula escamosa & 1 \\
\hline & Linfoma & 1 \\
\hline & Tumor neuroendocrino & 1 \\
\hline \multirow{4}{*}{$\begin{array}{l}\text { Sin } \\
\text { alteraciones }\end{array}$} & $\begin{array}{l}\text { Adenocarcinoma pulmonar } \\
\text { primario }\end{array}$ & 2 \\
\hline & Linfoma & 3 \\
\hline & Carcinoma de células pequeñas & 1 \\
\hline & $\begin{array}{l}\text { Metástasis de origen } \\
\text { ginecológico }\end{array}$ & 2 \\
\hline \multirow{2}{*}{$\begin{array}{l}\text { Compresión } \\
\text { extrínseca }\end{array}$} & $\begin{array}{l}\text { Adenocarcinoma pulmonar } \\
\text { primario }\end{array}$ & 1 \\
\hline & Carcinoma de células pequeñas & 1 \\
\hline $\begin{array}{l}\text { Disminución } \\
\text { del calibre }\end{array}$ & $\begin{array}{l}\text { Metástasis de origen } \\
\text { ginecológico }\end{array}$ & 1 \\
\hline
\end{tabular}

\section{DISCUSIÓN}

En la actualidad, las neoplasias pulmonares son consideradas una problemática de salud pública debido a su prevalencia y alta mortalidad. ${ }^{2}$ Por la historia natural de la enfermedad, el cáncer de pulmón se reconoce en estadíos avanzados. Desde el desarrollo del broncoscopio fibroóptico flexible como método diagnóstico para neoplasias pulmonares, ${ }^{11}$ se ha logrado mejorar el diagnóstico de neoplasias pulmonares hasta un $94 \%,{ }^{18}$ donde se conoce que hallazgos como zonas de eritema, pérdida de la anatomía bronquial y apariencia nodular de la mucosa sugiere altamente la presencia de neoplasia, la cual se debe confirmar histológicamente..$^{19}$

En el presente estudio se reconoció una tasa de acuerdo del $89 \%$, con un índice de acuerdo moderado, esto supera a los estudios previamente reportados que mencionan que aproximadamente $70 \%$ de neoplasias pulmonares son visibles en el estudio fibrobroncoscópico. Cabe recordar que este método es operador dependiente..$^{20-22}$

En los casos de neoplasia pulmonar maligna, el patrón más prevalente fue de adenocarcinoma pulmonar primario, seguido de carcinoma de célula escamosa. El rendimiento de la biopsia endobronquial es mayor para lesiones exofíticas visibles por fibrobroncoscopia, con un rendimiento diagnóstico aproximado del $90 \% .{ }^{23-26}$ Para los adenocarcinomas la tasa diagnóstica es del $52.6 \%$ y para los carcinomas de célula escamosa del $92 \%{ }^{18}$

Dentro de los casos con fibrobroncoscopia negativa para lesión tumoral y con histopatología positiva ( $\mathrm{n}=$ 22) (tabla 2), llama la atención los 4 casos de linfoma (1 caso de linfoma Hodgkin clásico tipo esclerosis nodular, 1 caso de linfoma B marginal, 1 caso de linfoma B difuso de alto grado y un caso de linfoma T extranodal NK), donde este hallazgo de ninguna lesión endoscópica o no relacionada con neoplasia se debe a que la infiltración bronquial suele ser submucosa y no sobresale por la vía aérea. ${ }^{27}$

El presente estudio mostró una concordancia moderada entre los hallazgos fibrobroncoscópicos e histopatológicos, con una tasa de acuerdo del 89\%. Estos hallazgos confirman que la sospecha en la endoscopia es fundamental para encaminar el diagnóstico y solicitar pruebas preliminares para un adecuado y oportuno estudio histológico.

El adenocarcinoma primario de pulmón fue el tumor que mostró menor acuerdo entre las dos pruebas, los 
hallazgos broncoscópicos fueron endobronquitis antracótica $(n=8)$, sin alteraciones $(n=2)$, y compresión extrínseca $(n=1)$; seguido de linfomas, en 3 casos no se observaron lesiones en la endoscopia y en 1 caso se reconoció endobronquitis antracótica. El tumor que mostró mayor acuerdo fue el carcinoma de célula escamosa y un caso de sarcoma de alto grado.

Desafortunadamente, en países como Colombia los trámites administrativos pueden hacer del proceso diagnóstico un camino largo y tortuoso, por lo que se vuelve indispensable crear estrategias locales rentables, eficaces, eficientes, multidisciplinarias, al alcance de los pacientes y con evidencia suficiente para adelantar y priorizar los estudios de detección temprana, caracterización y estratificación de cáncer de pulmón tales como marcadores de inmunohistoquímica y pruebas moleculares específicos basados en los hallazgos fibrobroncoscópicos e histológicos. ${ }^{28-31}$ Esto permitiría realizar un diagnóstico oportuno y por consiguiente dar un tratamiento dirigido en un menor tiempo.

\section{Conflicto de intereses}

Los autores declaran no tener conflicto de intereses.

\section{REFERENCIAS}

1. Welfare AloHa. Cancer in Australia: Actual incidence data from 1982 to 2013 and mortality data from 1982 to 2014 with projections to 2017. Asia Pac J Clin Oncol 2018;14(1):5-15. doi: 10.1111/ajco.12761.

2. Torre LA, Bray F, Siegel RL, Ferlay J, Lortet-Tieulent J, Jemal A. Global Cancer statistics, 2012. CA Cancer J Clin 2015;65(2):87-108. doi: 10.3322/caac.21262.

3. Humphrey LL, Deffebach M, Pappas M, et al. Screening for lung cancer with low-dose computed tomography: a systematic review to update the US Preventive services task force recommendation. Ann Intern Med 2013;159(6):411-420. doi: 10.7326/0003-4819-159-6201309170-00690.

4. Fritz I, Olsson H. Lung cancer in young women in southern Sweden: a descriptive study. Clin Respir J 2018;12(4):1565-1571. doi: 10.1111/crj.12712.

5. Hasan N, Kumar R, Kavuru MS. Lung cancer screening beyond low-dose computed tomography: the role of novel biomarkers. Lung 2014;192:639-648. doi: 10.1007/ s00408-014-9636-z.

6. Emery JD, Gray V, Walter FM, et al. The Improving Rural Cancer Outcomes (IRCO) Trial: a factorial cluster-randomised controlled trial of a complex intervention to reduce time to diagnosis in rural patients with cancer in Western Australia: a study protocol. BMJ Open 2014;4(9):e006156. doi: 10.1136/ bmjopen-2014-006156.

7. Zaric B, Perin B, Carapic V, et al. Diagnostic value of autofluorescence bronchoscopy in lung cancer.
Thorac Cancer 2013;4(1):1-8. doi: 10.1111/j.17597714.2012.00130.x.

8. Lam B, Wong MP, Fung SL, et al. The clinical value of autofluorescence bronchoscopy for the diagnosis of lung cancer. Eur Respir J 2006;28(5):915-919.

9. Labarca G, Aravena C, Ortega F, et al. Minimally invasive methods for staging in lung cancer: Systematic review and meta-analysis. Pulm Med 2016;2016:1024709.

10. Verma A, Kang YR, Kim H, Um SW. Ultrasound guided fine needle aspiration of a lung mass via a transesophageal approach using endobronchial ultrasound bronchoscope. Thorac Cancer 2012;3(3):280283. doi: 10.1111/j.1759-7714.2011.00090.x.

11. Ikeda S. Flexible bronchofiberscope. Ann Otol Rhinol Laryngol 1970;79(5):916-923.

12. Nahorecki A, Postrzech-Adamczyk K, Straszak E, Szuba A, Janczak D, Chabowski M. Effectiveness of bronchofiberoscopy in diagnosis of lung lesions. Adv Exp Med Biol 2017;980:11-17. doi: 10.1007/5584_2017_6.

13. Mino-Kenudson M. Immunohistochemistry for predictive biomarkers in non-small cell lung cancer. Transl Lung Cancer Res 2017;6(5):570-587. doi: 10.21037/tlcr.2017.07.06.

14. Szutowicz E, Dziadziuszko R. Quantitative immunohistochemistry in lung cancer: clinical perspective. Folia Histochem Cytobiol 2010;48(1):7-11. doi: 10.2478/v10042-010-0014-2.

15. Donner A, Eliasziw M. Sample size requirements for reliability studies. Stat Med 1987;6(4):441-448.

16. Landis JR, Koch GG. The measurement of observer agreement for categorical data. Biometrics 1977;33(1):159-174.

17. Travis WD, Brambilla E, Burke AP, Marx A, Nicholson AG. WHO classification of tumours of the lung, pleura, thymus and heart. 4th ed. WHO. 2015.

18. Arroliga AC, Matthay RA. The role of bronchoscopy in lung cancer. Clin Chest Med 1993;14(1):87-98.

19. Mazzone P, Jain P, Arroliga AC, Matthay RA. Bronchoscopy and needle biopsy techniques for diagnosis and staging of lung cancer. Clin Chest Med 2002;23(1):137-158, ix.

20. El-Bayoumi E, Silvestri GA. Bronchoscopy for the diagnosis and staging of lung cancer. Semin Respir Crit Care Med 2008;29(3):261-270. doi: 10.1055/s-2008-1076746.

21. Govert JA, Dodd LG, Kussin PS, Samuelson WM. A prospective comparison of fiberoptic transbronchial needle aspiration and bronchial biopsy for bronchoscopically visible lung carcinoma. Cancer 1999;87(3):129-134.

22. de Gracia J, Bravo C, Miravitlles M, et al. Diagnostic value of bronchoalveolar lavage in peripheral lung cancer. Am Rev Respir Dis 1993;147(3):649-652.

23. Nair CK, Mathew AP, George PS. Lung cancer: presentation and pattern of care in a cancer center in South India. Indian J Cancer 2017;54(1):164-168. doi: 10.4103/ijc.IJC_56_17.

24. Wilson RW, Frazier AA. Pathological-radiological correlations: pathological and radiological correlation of endobronchial neoplasms: part II, malignant tumors. Ann Diagn Pathol 1998;2(1):31-34. 
25. Simoff MJ. Endobronchial management of advanced lung cancer. Cancer Control 2001;8(4):337-343.

26. Schreiber G, McCrory DC. Performance characteristics of different modalities for diagnosis of suspected lung cancer: summary of published evidence. Chest 2003;123(1 Suppl):115s-128s.

27. William J, Variakojis D, Yeldandi A, Raparia K. Lymphoproliferative neoplasms of the lung: a review. Arch Pathol Lab Med 2013;137(3):382-391. doi: 10.5858/ arpa.2012-0202-RA.

28. Bhargava A, Bunkar N, Aglawe A, et al. Epigenetic biomarkers for risk assessment of particulate matter associated lung cancer. Curr Drug Targets
2018;19(10):1127-1147. doi: 10.2174/138945011866617 0911114342.

29. Pezzuto A, Terzo F, Graziani ML, Ricci A, Bruno P, Mariotta $S$. Lung cancer requires multidisciplinary treatment to improve patient survival: a case report. Oncol Lett 2017;14(3):3035-3038. doi: 10.3892/ol.2017.6511.

30. Salomaa ER, Sällinen S, Hiekkanen H, Liippo K. Delays in the diagnosis and treatment of lung cancer. Chest 2005;128(4):2282-2288.

31. Verma A, Lim AY, Tai DY, et al. Timeliness of diagnosing lung cancer: number of procedures and time needed to establish diagnosis: Being right the first time. Medicine (Baltimore) 2015;94(29):e1216. doi: 10.1097/MD.0000000000001216. 